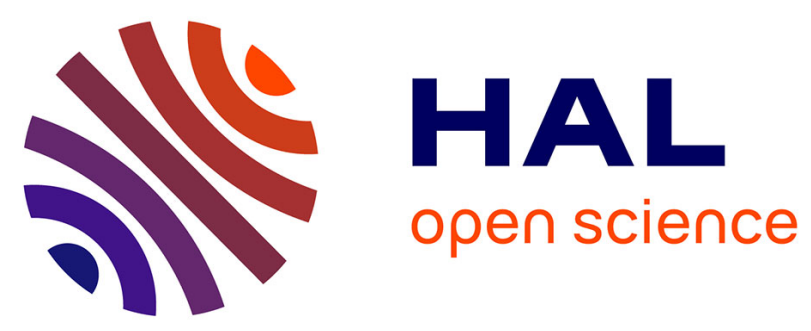

\title{
REVISITING THE MARKOV CHAIN MODEL OF IEEE 802.11E EDCA AND INTRODUCING THE VIRTUAL COLLISION PHENOMENON
}

\author{
Mohamad El Masri, Guy Juanole, Slim Abdellatif
}

\section{- To cite this version:}

Mohamad El Masri, Guy Juanole, Slim Abdellatif. REVISITING THE MARKOV CHAIN MODEL OF IEEE 802.11E EDCA AND INTRODUCING THE VIRTUAL COLLISION PHENOMENON. International Conference on Wireless Information Networks and Systems, Jul 2007, Barcelone, Spain. pp.76-84. hal-00389415

\section{HAL Id: hal-00389415 https://hal.science/hal-00389415}

Submitted on 28 May 2009

HAL is a multi-disciplinary open access archive for the deposit and dissemination of scientific research documents, whether they are published or not. The documents may come from teaching and research institutions in France or abroad, or from public or private research centers.
L'archive ouverte pluridisciplinaire $\mathbf{H A L}$, est destinée au dépôt et à la diffusion de documents scientifiques de niveau recherche, publiés ou non, émanant des établissements d'enseignement et de recherche français ou étrangers, des laboratoires publics ou privés. 


\title{
REVISITING THE MARKOV CHAIN MODEL OF IEEE 802.11E EDCA AND INTRODUCING THE VIRTUAL COLLISION PHENOMENON Preparation of Camera-Ready Contributions to INSTICC Proceedings*
}

\author{
Mohamad El Masri ${ }^{\circ}$, Guy Juanole ${ }^{\ddagger}$, Slim Abdellatif ${ }^{\circ}$ \\ LAAS-CNRS; ${ }^{\circ}$ INSA, ${ }^{\ddagger}$ UPS; University of Toulouse, Toulouse, France \\ masri@laas.fr, juanole@laas.fr, slim@laas.fr
}

\begin{abstract}
Keywords: $\quad$ Quality Of Service, Wireless Local Area Networks, Modelling, Markov Chain
Abstract: $\quad$ IEEE 802.11e specifies the Enhanced Distributed Channel Access (EDCA) for distributed access with traffic differentiation to a Wireless Local Area Network (WLAN). Traffic differentiation led to introducing several traffic queues within each station, thus introducing virtual collision. In this paper, we present a pattern based Markov chain model of EDCA including the virtual collision phenomenon not found in previous work. We also correct the misconceptions found in existing Markov chain models of EDCA (Kong et al., 2004). We thus obtain a more realistic model of IEEE 802.11e EDCA which will allow a more precise performance analysis that may be used for admission control.
\end{abstract}

\section{INTRODUCTION}

The IEEE 802.11e work group (802.11e, 2005) introduced QoS (Quality of Service) mechanisms into the MAC layer (Medium Access Control) of the legacy IEEE 802.11 standard. This mainly consisted in the definition of a new access function: HCF (Hybrid Access Control) which combines two access modes, one of these is EDCA (Enhanced Distributed Channel Access) which is an enhancement of DCF (Distributed Coordination Function based on a CSMA/CA scheme). EDCA is the area of interest of our work. With respect to DCF, EDCA introduces the traffic differentiation concept, thus defining four access categories $(A C)$, each corresponding to a different queue within the station. A CSMA/CA scheme is implemented by each $A C$. This scheme is based on the arbitration (characterized by the AIFS parameter (Arbitration Inter Frame Space)) and on the backoff procedure (characterized by the contention window (CW) and the range $\left.\left[C W_{\min }, C W_{\max }\right]\right)$. AIFS and $\mathrm{CW}$ play the same role as DIFS and CW in DCF. The choice of AIFS and CW allow to prioritize the $A C$ traffic (the smaller the AIFS and CW, the higher the access probability). Due to the presence of several queues within a station, EDCA introduces, in addition to real collisions (physical collisions in the channel involving

\footnotetext{
*This work is partially funded by both EuQoS and SATSIX projects
}

queues from different stations), a new kind of collisions, named virtual collisions. These latter take place when at least two queues from the same station try to access the medium at the same time after their backoff period. It results in granting the access to the highest priority queue and penalizing the others (by widening the $\mathrm{CW}$ in the same manner as a real collision). EDCA involves several complex mechanisms, those are not always clearly specified thus inducing a lot of questioning among the people working on it. The use of formal methods is of great interest to help the understanding and guide the analysis and the performance evaluation of the protocol. Some models have already been proposed in the literature (Zhu and Chlamtac, 2005; Kong et al., 2004; Wu et al., 2006), models which assume a saturation regime (thus frequent collisions) and which are mainly inspired by the Bianchi model of DCF (Bianchi, 2000). Zhu and Chlamtac (Zhu and Chlamtac, 2005) proposed a two dimensional model of an EDCA $A C$ which considers neither the virtual collision aspect nor the time elapsing during a transmission on the medium. The model of Kong et. al. (Kong et al., 2004) captures this latter aspect (at a cost of a new dimension in the model - the overall model of EDCA is a three dimensional discrete Markov chain). However it does not describe explicitly the virtual collision nor does it represent all the mechanisms described in the standard (802.11e, 2005; Mangold et al., 2003). Wu et. al.'s approach 
(Wu et al., 2006) of the different contention zones is novel, but the consequences of a virtual collision are not clearly detailed.

The goal of this work is, based on the model of Kong et. al.(Kong et al., 2004), to define, in a pedagogical way, a model of an EDCA $A C$, as complete as possible i.e. which integrates all the mechanisms specified by the standard and which to the best of our knowledge has never been done. This model is introduced progressively by identifying the main behavioral patterns of the EDCA $A C$.

The paper, dedicated mainly to a careful modelling, is organized as follows: first we define a modelling methodology, we then give the models of the different patterns and the global model. We then expose the formulas, which can be gotten from the model and which are the basics for performance analysis. A validation of the model in different cases is detailed in the following section. We then conclude by giving the perspectives of this work.

\section{GUIDES FOR THE MODELLING}

\section{1 $A C_{i}$ behavioral view}

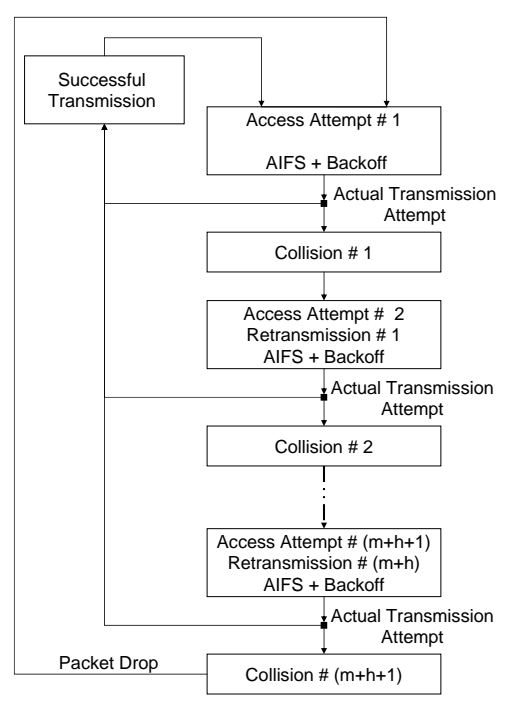

Figure 1: Behavior of an $A C_{i}$

We give an abstract view of an $A C_{i}$ 's behavior ( $i \in$ $(0,1,2,3)$ in descending priority order) in figure 1 . The transmission of a packet is implemented through a series of access attempts. Each is based, at first, on the sequence of two processes (AIFS and backoff), defining the medium idleness test before the actual transmission attempt, and then on the actual trans- mission attempt (i.e. the decision to make a transmission). The result of each actual transmission is either a successful transmission (following which the sending of a new packet is considered) or a collision (following which the packet's retransmission is considered). Note that on the first attempt we have $C W\left[A C_{i}\right]=C W_{\min }\left[A C_{i}\right]$. After a collision situation, the new value of the contention window is computed as follows:

$C W_{\text {new }}\left[A C_{i}\right]=\min \left(2 * C W\left[A C_{i}\right]+1, C W_{\text {max }}\left[A C_{i}\right]\right)$ in order to try to avoid further collisions. We define $m$ so that after $m$ transmissions, the value of $C W\left[A C_{i}\right]$ is $C W_{\max }\left[A C_{i}\right], h$ is the additional number of retransmissions needed before reaching the Retransmission Threshold $\left(R_{-} T\left[A C_{i}\right]=m+h\right)$. If the retransmission threshold is reached, the packet is dropped and the transmission of a new packet is considered. This $a b-$ stract view highlights the basic patterns for the modelling: AIFS procedure, Backoff procedure, actual transmission attempt procedure and their results.

\subsection{The basic patterns}

\subsubsection{AIFS procedure}

Any transmission attempt starts with the random choice of the value of the Backoff Counter $\left(B_{-} C\left[A C_{i}\right]\right)$ within the current contention window range $\left[0, C W\left[A C_{i}\right]\right]$ (this value defines the backoff time which will be used at the end of the AIFS period). The AIFS procedure consists in the necessity to observe the medium idleness during the AIFS period. If, during the AIFS period (We call $A$ its duration in terms of time slots), the medium becomes busy, we have the AIFS decrementing freeze during the medium occupation time (we call $N$ the mean value of this duration in terms of time slots) after which the AIFS countdown is reset. At the end of the last slot of AIFS, if the medium is still idle, two outputs are possible: if $B_{-} C\left[A C_{i}\right]=0$, the $A C$ will directly attempt a transmission; if $B_{-} C\left[A C_{i}\right]>0$, the value of $B_{-} C\left[A C_{i}\right]$ is decremented of one, thus initiating the backoff procedure.

\subsubsection{Backoff procedure}

A backoff procedure will mainly consist in decrementing the value of $B_{-} C\left[A C_{i}\right]$ while the medium is idle. The value of $B_{-} C\left[A C_{i}\right]$ is decremented until it reaches 0 , one slot after which a transmission is directly attempted if the medium is still idle. If during the backoff counter decrementing, the medium becomes busy, the decrementing procedure is stopped and frozen during a time which is the sum of the medium occupation time and an AIFS period (this time has the value $N+A$ ), if during the AIFS period, the medium is busy again, the process is repeated. At the end of the last slot of AIFS, if the medium is still idle, two outputs are possible: if $B_{-} C\left[A C_{i}\right]=0$, the $A C$ 
will directly attempt a transmission; if $B_{-} C\left[A C_{i}\right]>0$, the value of $B_{-} C\left[A C_{i}\right]$ is decremented, thus resuming the backoff procedure.

\subsubsection{Actual transmission attempt}

When an $A C_{i}$ decides to initiate a transmission attempt, either it is the only one within the station to want to transmit, in which case it will directly access the medium, or there is at least another $A C$ within the station also wishing to transmit, in which case both $A C$ s will go into a virtual collision. Within the virtual collision handler, the $A C$ winner of the virtual collision (thus accessing the medium) is the higher priority $A C$. If $A C_{i}$ loses the virtual collision, then the medium will be accessed by an $A C$, virtually colliding with $A C_{i}$ and having a higher priority. An actual transmission attempt is followed by three outcomes:

1. The transmission was successful, in which case $A C_{i}$ occupied the medium for a duration $\left\lceil T_{S}\right\rceil\left(\left\lceil T_{S}\right\rceil\right.$ is the smallest integer -in time slots- higher than $T_{S}$ the duration of a successful transmission) and a new packet transmission is then taken into consideration.

2. $A C_{i}$ suffered a real collision, in which case $A C_{i}$ occupied the medium for a collided transmission time $\left\lceil T_{c}\right\rceil$ and the packet may be retransmitted within the retry threshold limit.

3. $A C_{i}$ lost a virtual collision, in which case $A C_{i}$ will not occupy the medium, a higher priority $A C$ within the station will transmit (either suffering a collision thus occupying the medium for $\left\lceil T_{c}\right\rceil$ or transmitting successfully thus occupying the medium for $\left.\left\lceil T_{S}\right\rceil\right)$. $A C_{i}$ 's packet may be retransmitted within the retry threshold limit.

Situations 2 and 3 above define globally, what we call, the collision situation for $A C_{i}$.

\section{$3 A C_{I}$ MODELLING}

\subsection{Basics for the modelling}

$A C_{i}$ Behavior: We represent it by a discrete Markov chain where the state must be represented without ambiguity. A state of the discrete Markov chain must specify both the packet access attempts (we have to distinguish on one hand the successive attempts and their corresponding collisions and on the other hand a successful transmission), the backoff counter (we have to distinguish on one hand the backoff procedure where the backoff counter is meaningful and on the other hand the situations where the backoff counter is meaningless) and the remaining time to the end of the different timed actions (AIFS, medium occupancy, collision, successful transmission). Therefore a state of the discrete Markov chain is represented by a triplet $(j, k, d)$ with $j$ representing the state of the packet attempt, $k$ the backoff counter and $d$ the remaining time. We consider the following values for each of the components:

- $j: 0 \leq j \leq m+h$ for the successive attempts $(j=0$ for the first attempt and $1 \leq j \leq m+h$ for the following retransmission attempts), each value of $j$ is associated to all the states of the AIFS period before the backoff, the stage of the backoff procedure where the value of the contention window $C W\left[A C_{i}\right]$ is noted $W_{j}$, and the collision situation; the successful transmission is represented by $j=-1$.

- $k$ : $0 \leq k \leq W_{j}$ for stage $j$ of the backoff procedure; in the other cases where $k$ is meaningless we take a negative value for $k$ (different negative values should be taken, for triplet uniqueness reasons, depending on the situation as we explain after the specification of the values of $d$ ).

- $d: 1 \leq d \leq\left\lceil T_{S}\right\rceil$ for the duration of a successful transmission of $A C_{i}$ or after a virtual collision of $A C_{i}$ (where $A C_{k}$, winner of the virtual collision, successfully transmits); $1 \leq d \leq\left\lceil T_{c}\right\rceil$ for the duration of a collision (of either $\bar{A} C_{i}$ or $A C_{k}$ winner of the virtual collision); $1 \leq d \leq A$ for the AIFS duration; $A+1 \leq d \leq N+A$ for the medium occupancy duration occurring during an AIFS period or during backoff counter decrementing. Note that $\left\lceil T_{c}\right\rceil<\left\lceil T_{S}\right\rceil$.

As for each attempt $j$ the AIFS period before the backoff and the collision situation (in both situations the backoff counter is meaningless) can have remaining time values which can be identical, it is necessary, in order to avoid state ambiguity, to distinguish these states by a different negative value of $k$; we choose: $k=-1$ for the collision situation and $k=-2$ for the AIFS period. The value of $k$ for the successful transmission period is not problematic because of the different value of $j$, we thus choose $k=-1$.

Transition probabilities: Before defining the different pattern models forming the whole model, we must define the probabilities that will be associated to the transitions. At first we define the following basic probabilities:

- The probability related to the fact that the medium is busy $\left(p_{b}\right)$ or not $\left(1-p_{b}\right)$.

- The probabilities related to the access attempt of $A C_{i}$, whether competing or not with the other access categories within the station (leading in the first case to a virtual collision situation): $\overline{p_{i}^{v}}$ is the probability for $A C_{i}$ not to go into a virtual collision when attempting to access, $p_{i}{ }^{w v}$ is the probability for $A C_{i}$ to go into a virtual collision and win it and $p_{i}^{l v}$ is the probability for $A C_{i}$ to go into a virtual collision and lose it. Note that $\overline{p_{i}^{v}}+p_{i}{ }^{w v}+p_{i}{ }^{l v}=1$. 
- The probability for $A C_{i}$ to suffer a real collision during its actual access to the medium (i.e. either $A C_{i}$ went into a virtual collision and won it or did not go into a virtual collision at all): $p_{i}^{r}$. We have $p_{i}^{r}+\overline{p_{i}^{r}}=1$.

- The probability (after the loss of a virtual collision by $A C_{i}$ ) for the $A C$ winning the virtual collision (let $A C_{k}$ be it) to suffer a real collision: $p_{k}^{r}$. We have $p_{k}^{r}+\overline{p_{k}^{r}}=1$.

- The probability of the random choice of the Backoff Counter $\left(B_{-} C\left[A C_{i}\right]\right)$ within the contention window for the $j^{\text {th }}$ retransmission is $\frac{1}{W_{j}+1}$.

Based on those basic probabilities, we define the probabilities characterizing the collision situation:

- $p_{i}^{(2)}$ is the probability of an unsuccessful transmission attempt resulting in a $\left\lceil T_{c}\right\rceil$ slot occupation of the medium, i.e. either $A C_{i}$ suffered a real collision or $A C_{i}$ lost a virtual collision and $A C_{k}$ winner of this virtual collision suffers a real collision: $p_{i}^{(2)}=\left(\overline{p_{i}^{v}}+p_{i}{ }^{w v}\right) p_{i}^{r}+p_{i}^{l v} p_{k}^{r}$.

- $p_{i}^{(3)}$ is the probability of an unsuccessful transmission attempt resulting in a $\left\lceil T_{s}\right\rceil$ slot occupation of the medium, i.e. $A C_{i}$ loses a virtual collision and $A C_{k}$, winner of the virtual collision, successfully transmits: $p_{i}^{(3)}=p_{i}^{l v} \overline{p_{k}^{r}}$.

- $p_{i}$ is the probability of a collision of $A C_{i}$ (a real collision or a lost virtual collision): $p_{i}=p_{i}^{(2)}+$ $p_{i}^{(3)}$.

\subsection{Models of the basic patterns}

We at first present the graphs of each model, we then indicate how to get the global model from these graphs. In each of the following models we represent the input and output states in bold line type and the internal states in normal line type. The states that do not belong to the presented pattern (which either lead to an input state of the pattern or are led to from an output state) are represented in dotted line type (note that those external states are necessarily output/input states of other patterns). All the transitions are labelled with the transition probabilities presented in section 3.1.

\subsubsection{Pattern: AIFS procedure and outputs}

The model is given in figure 2 . The different states of the pattern are self explanatory. We added to each of the transitions from the output state $(j,-2,1)$ a Predicate/Transition type label. The predicate is the value of the Backoff Counter $\left(B_{-} C\left[A C_{i}\right]\right)$ that has been randomly chosen at the beginning of the AIFS procedure (see section 2.2.1). If $B_{-} C\left[A C_{i}\right]=0$, there will be a transmission attempt at the end of the last slot of AIFS if the medium is still idle, the transmission attempt will either lead to a successful transmission (state $\left(-1,-1,\left\lceil T_{S}\right\rceil\right)$ ) or to a collision (state $\left(j,-1,\left\lceil T_{s}\right\rceil\right)$ in case $A C_{i}$ loses a virtual collision and $A C_{k}$, winner of the virtual collision, transmits successfully, or state $\left(j,-1,\left\lceil T_{c}\right\rceil\right)$ in case $A C_{i}$ collides or in case it loses a virtual collision and $A C_{k}$ collides). If $B_{-} C\left[A C_{i}\right]>0$, the chain transits into one of the states $\left[(j, 0,0),(j, 1,0) \ldots\left(j, W_{j}-1,0\right)\right]$ representing the beginning of the backoff procedure.

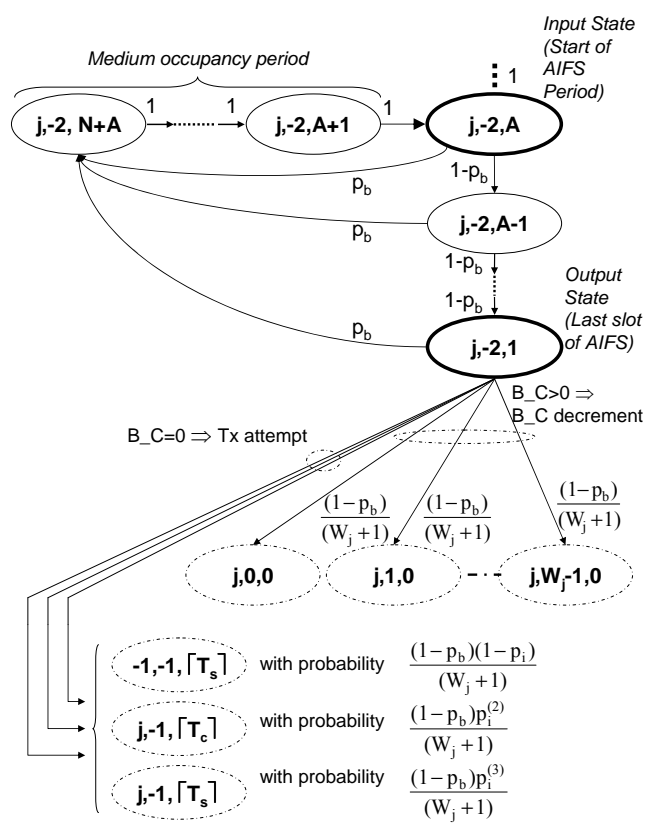

Figure 2: AIFS procedure pattern: $0 \leq j \leq m+h$

\subsubsection{Pattern: Backoff procedure and outputs}

The model is given in figure 3 . The input states of the model are $\left[(j, 0,0),(j, 1,0) \ldots\left(j, W_{j}-1,0\right)\right]$. The transitions between these states represent the decrementing of the backoff counter while the medium is idle (probability $1-p_{b}$ ). If the medium goes busy (probability $p_{b}$ ), the decrementing will be frozen during the medium occupancy and an AIFS period (represented by the subset of states above each counter decrementing state). From the output states $((j, 0,0)$ or $(j, 0,1))$, a transmission is attempted if the medium is idle. The transmission attempt will lead into one of the states $\left(-1,-1,\left\lceil T_{S}\right\rceil\right),\left(j,-1,\left\lceil T_{S}\right\rceil\right),\left(j,-1,\left\lceil T_{c}\right\rceil\right)$ (as in section 3.2.1 - case where $B_{-} C\left[A C_{i}\right]=0$ ).

\subsubsection{Pattern: Actual transmission attempt}

The model is given in figure 4 . The states $(j,-2,1)$, $(j, 0,1)$ and $(j, 0,0)$ are respectively the output states in the model "AIFS Procedure" for the first one and "Backoff procedure" for the two others. Those are the 


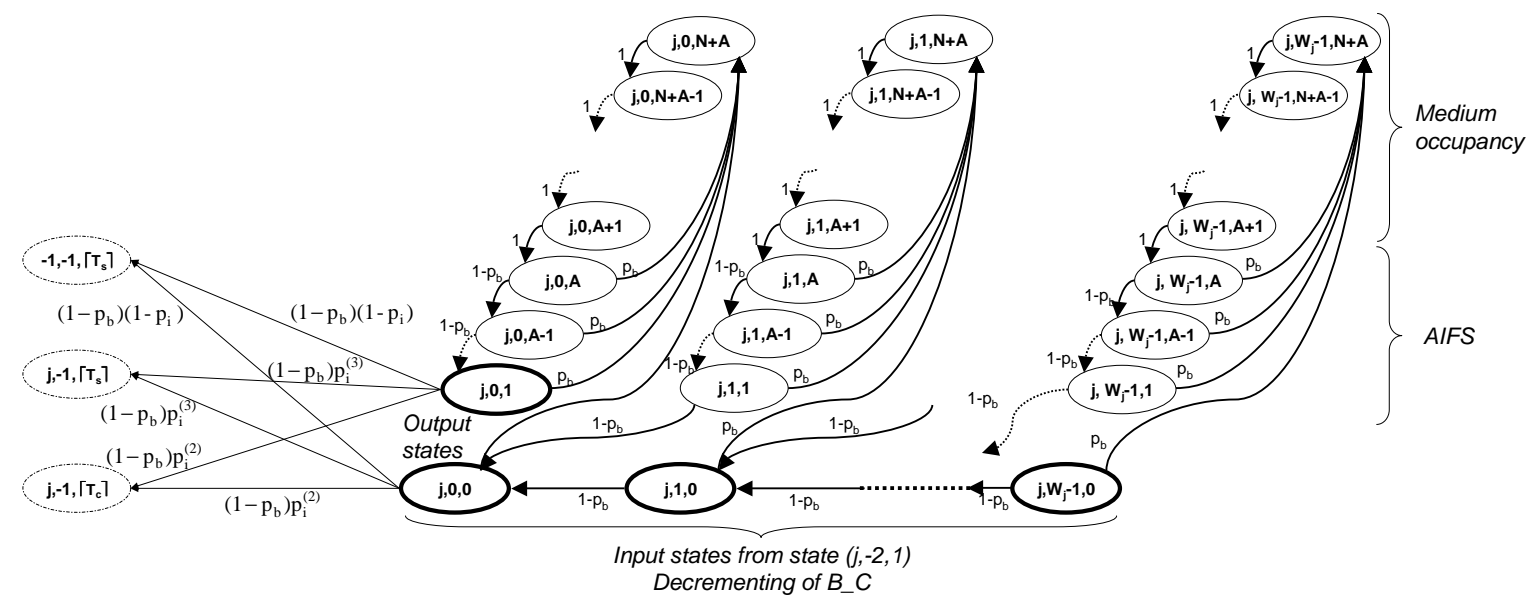

Figure 3: Backoff Procedure pattern: $0 \leq j \leq m+h$

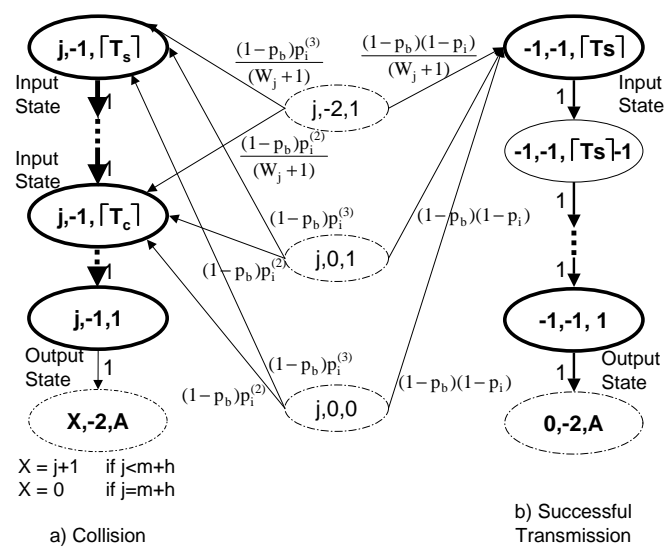

Figure 4: Outcomes of an actual transmission attempt: $0 \leq j \leq m+h$

states leading to a transmission attempt and resulting in either a successful transmission (right part of the figure) or a collision (left part of the figure). In case of a collision, two different entry states are possible (both leading to state $(j,-1,1)$ meaning two different medium occupancy time):

- states $\left(j,-1,\left\lceil T_{s}\right\rceil\right)$ for a $\left\lceil T_{s}\right\rceil$ occupancy time in case $A C_{i}$ lost a virtual collision and $A C_{k}$, winner of the virtual collision, successfully transmits;

- $\left(j,-1,\left\lceil T_{c}\right\rceil\right)$ for a $\left\lceil T_{c}\right\rceil$ occupancy time either in case $A C_{i}$ accesses the medium and collides or in case $A C_{i}$ loses a virtual collision and $A C_{k}$, winner of the virtual collision, collides.

Once the process is finished it will lead:

- in case of a successful transmission, a new packet is taken into consideration, we thus go to its first access attempt (state $(0,-2, A)$ );

- in case of a collision, if the retry threshold has not been reached, the packet will go into a new transmission attempt (state $(j+1,-2, A))$, if the retry threshold has been reached, the packet is dropped and a new packet is taken into consideration (state $(0,-2, A))$.

\subsection{Global model}

The global model is got by connecting the models of the different "Access Attempts" following the guide of figure 1 (with $j=0,1,2 \ldots m, \ldots m+h$ ).

\section{CRITICISM OF KONG ET. AL. MODEL}

Several points of the behavior of an AC are not represented in Kong et. al.'s model (Kong et al., 2004):

- In Kong et. al.'s model, an AIFS period is considered only for the first transmission attempt (i.e. before the backoff procedure of the first attempt). However, EDCA stipulates also an AIFS period before the launch of every retransmission's backoff period. This is considered in our model with pattern AIFS in figure 2 covering the first attempt $(j=0)$ and the retransmissions $(j>0)$.

- In EDCA, when the randomly chosen value of the backoff counter is 0 , a transmission can be attempted, if the medium is idle, directly after the last slot of AIFS. This is not represented in Kong et. al.'s model. In figure 4, this aspect is modelled by introducing the possibility of attempting a transmission after the last slot of AIFS when the backoff counter is zero (in states $(j,-2,1)$ and $(j, 0,1))$ in addition to state $(j, 0,0)$ considered by Kong et. al..

- The virtual collision does not appear explicitly in Kong et. al.'s model. This leads to erroneous representation of the collision (considering both types of collision as effective accesses 
to the medium which is not true). By integrating a correct representation of the virtual collision to the model (figure 4), we achieve a more precise model.

- Before attempting a medium access, an $A C$ should check for medium idleness, this is not represented in Kong et. al.'s model. In our model, transmission attempts (from states $(j,-2,1),(j, 0,1)$ and $(j, 0,0))$ are made with probability $\left(1-p_{b}\right)$ i.e. with a medium idle condition.

\section{FUNDAMENTAL RELATIONS FOR PERFORMANCE ANALYSIS}

\subsection{Steady state probability}

Let $b_{j, k, d}$ be the steady state probability of state $(j, k, d)$ defined earlier in section 3.1. We derived for each of the patterns described in section 3.2 a set of equations expressing the steady state probabilities of each state in terms of $b_{0,-2,1}$. We chose this state because it is the first state from which an access may be attempted. With those equations and the normalization condition we obtain the expression of all the probabilities in terms of $m, h, A,\left\lceil T_{s}\right\rceil,\left\lceil T_{c}\right\rceil, N$ and $W_{j}$ which are known and $, p_{i}, p_{i}^{(3)}$ and $p_{b}$ which will be expressed in the following section. For lack of space we only give the expression of $b_{0,-2,1}$, all other steady state probabilities can be easily found using classical methods of steady state analysis. We have:

$$
\begin{aligned}
b_{0,-2,1}= & {\left[\left\lceil T_{S}\right\rceil\left(1-p_{b}\right)\left(1-p_{i}^{m+h+1}\right)\right.} \\
& +p_{i}\left\lceil T_{C}\right\rceil\left(1-p_{b}\right) \frac{1-p_{i}^{m+h+1}}{1-p_{i}} \\
& +p_{i}^{(3)}\left(\left\lceil T_{s}\right\rceil-\left\lceil T_{c}\right\rceil\right)\left(1-p_{b}\right) \frac{1-p_{i}^{m+h+1}}{1-p_{i}} \\
& +\frac{1+N p_{b}}{p_{b}} \frac{1-\left(1-p_{b}\right)^{A}}{\left(1-p_{b}\right)^{A-1}} \frac{1-p_{i}^{m+h+1}}{1-p_{i}} \\
& \left.+\frac{1+N p_{b}}{2\left(1-p_{b}\right)^{A-1}} \sum_{j=0}^{m+h} p_{i}^{j} W_{j}\right]^{-1}
\end{aligned}
$$

This formula, if compared to Kong et. al.'s formula, points out the main differences between both models: the single $\left(1-p_{b}\right)$ factor in the first three terms of the sum indicates the idleness test before an access is attempted; introducing an additional term to the sum representing the different outcomes of a collision (third term of the sum); $\frac{1-p_{i}^{m+h+1}}{1-p_{i}}$ factor (which in fact is equivalent to $\left.\sum_{j=0}^{m+h} p_{i}^{j}\right)$ in the fourth term of the sum corresponding to the AIFS arises from the fact our model considers (as specified earlier) the AIFS periods after a collision and so on.

\subsection{Collision probability and busy probability}

We give in the following sections the expressions of the different probabilities used in the model. These are essential for the use of the model for any performance analysis. We differentiate three aspects of the $A C$ behavior: access attempt, actual access and medium occupation. Unlike Kong et. al. we differentiate an access attempt from an actual access, an access attempt describes the possible contention between different $A C$ s within a station. Thus an access attempt followed by a lost virtual collision will not result in an actual access.

\subsubsection{Access attempt}

Let $\alpha_{i}$ be the probability for $A C_{i}$ to attempt a transmission in a randomly chosen time slot. $\alpha_{i}$ can thus be written:

$$
\alpha_{i}=\left(1-p_{b}\right) \sum_{j=0}^{m+h}\left(\frac{1}{W_{j}+1} b_{j,-2,1}+b_{j, 0,1}+b_{j, 0,0}\right)
$$

The virtual collision probabilities can be written:

- $\overline{p_{i}^{v}}=\prod_{x \in[0 . .3] x \neq i}\left(1-\alpha_{x}\right)$

- $p_{i}^{l v}=0$ if $i=0$

- $p_{i}^{l v}=1-\prod_{x<i}\left(1-\alpha_{x}\right)$ otherwise

- $p_{i}{ }^{w v}=1-\left(p_{i}^{l v}+\overline{p_{i}^{v}}\right)$

\subsubsection{Actual access}

We define $\tau_{i}$ (respectively $\tau$ ) as the probability for $A C_{i}$ (respectively a station of the network) to effectively access the medium at a randomly chosen time. We have:

$$
\tau_{i}=\left(\overline{p_{i}^{v}}+p_{i}^{w v}\right) \alpha_{i} \text { and } \tau=\sum_{i=0}^{i=3} \tau_{i}
$$

Thus we may define $p_{i}^{r}$ (the real collision probability of $A C_{i}$ ) as the probability for at least two stations to access the medium in a randomly chosen time slot: $p_{i}^{r}=1-(1-\tau)^{M-1}(M$ being the number of stations in the network), the same applies to the value of $p_{k}^{r}$. These definitions allow us to write $p_{i}, p_{i}^{(2)}$ and $p_{i}^{(3)}$ defined in section 3.1 .

\subsubsection{Medium occupation}

In order to define the busy probability, we define $v_{i}$ (respectively $v$ ) as the probability that the channel is occupied by a given $A C_{i}$ (respectively a station) in a given time slot. $v_{i}$ can in fact be understood as the probability for the Markov chain to be in one of the states where an access is ongoing (a successful transmission or a real collision of $\left.A C_{i}\right)$. We thus have:

$$
v_{i}=\sum_{d=1}^{\left\lceil T_{s}\right\rceil} b_{-1,-1, d}+\frac{\left(\overline{p_{i}^{v}}+p_{i}{ }^{w v}\right) p_{i}^{r}}{p_{i}^{(2)}+p_{i}^{(3)}} \sum_{j=0}^{m+h} \sum_{d=1}^{\left\lceil T_{c}\right\rceil} b_{j,-1, d}
$$


and $v=\sum_{i=0}^{i=3} v_{i}$. The channel busy probability $p_{b}$ can thus be expressed as the probability that at least one station occupies the medium (complementary of the probability that no station occupies the medium): $p_{b}=1-(1-v)^{M}$.

\subsection{Throughput}

Let $S$ be the normalized throughput as defined by Bianchi (Bianchi, 2000): $S$ is the ratio of time the channel is used to transmit payload bits. Further on we define $s_{i}$, the normalized throughput of $A C_{i}$ as the ratio of time the channel is used to transmit payload bits corresponding to an $A C_{i}$ of a station, and $S_{i}$ as the normalized throughput of all $A C_{i}$ s of the same priority in the network. We use for the throughput calculation the formula described by Kong et. al. in (Kong et al., 2004).

$$
S_{i}=\frac{p_{s i} E[P]}{E[I]+\sum_{i^{\prime}=0}^{3} p_{s i^{\prime}}\left(T_{s}+A I F S\left[A C_{i^{\prime}}\right]\right)+\left(1-\sum_{i^{\prime}=0}^{3} p_{s i^{\prime}}\right) T_{c}}
$$

with $\mathrm{E}[\mathrm{P}]$ being the mean payload transmission time in slot times, $\mathrm{E}[\mathrm{I}]$ the mean idle period obtained as $E[I]=\frac{1}{p_{b}}-1$ and $p_{s i}$ the conditional transmission probability:

$$
p_{s i}=\frac{M\left((1-v)^{(M-1)}\right)\left(\prod_{i^{\prime}>i}\left(1-v_{i^{\prime}}\right)\right)\left(\sum_{d=1}^{\left[T_{S}\right\rceil} b_{-1,-1, d}\right)}{1-(1-v)^{M}}
$$

\section{MODEL VALIDATION}

The Markov Chain presented in section 3 represents the behavior of an $A C_{i}$ in a local context, that of the station the $A C_{i}$ is in; and a global context, that of the different stations in the network. These contexts are integrated to the model using a number of parameters: the different collisions probabilities $p_{i}(2)$ and $p_{i}(3)$ and the busy probability $p_{b}$. Two different uses of the model are investigated hereafter:

- as a formula based model: using the formulas presented in sections 5.2 to represent the contexts in which evolves $A C_{i}$.

- as a measurement based model, replacing the above mentioned formulas with measures of the state of both the network and the local context.

In order to confirm the behavior of the model, a set of simulations was carried out. We detail in the following section the simulation set, we then measure up the results given by the model in both its use cases to the results obtained by simulation (from a throughput point of view).

\subsection{Simulation Set}

M EDCA enabled stations share a medium. The stations' physical bit rate is set to $11 \mathrm{Mbps}$. In each station the categories AC_VO, AC_VI and AC_BE

\begin{tabular}{|c|c|}
\hline Variable & Value \\
\hline $\mathrm{M}$ (number of stations) & $2 . .9$ \\
\hline Payload size & 1000 bytes \\
\hline$\left\lceil T_{S}\right\rceil$ & 55 \\
\hline$\left.T_{C}\right\rceil$ & 14 \\
\hline AIFS AC_VO & 2 \\
\hline AIFS AC_VI & 2 \\
\hline AIFS AC_BE & 3 \\
\hline$C W$ range AC_VO & $3 . .7$ \\
\hline$C W$ range AC_VI & $3 . .15$ \\
\hline$C W$ range AC_BE & $15 . .1023$ \\
\hline Retry Limit & 4 \\
\hline
\end{tabular}

Table 1: Details of variables used for simulation

of each station are saturated with 1000 byte payload packets. RTS-CTS handshake is enabled. The technical details of the simulations (used also for the model based performance analysis) are given in table 1 .

\subsection{Formula based use of the model}

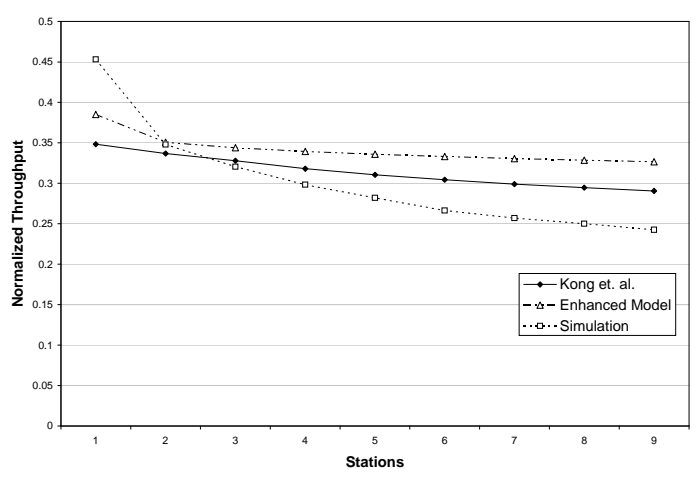

Figure 5: Comparing the Throughput of AC_VO

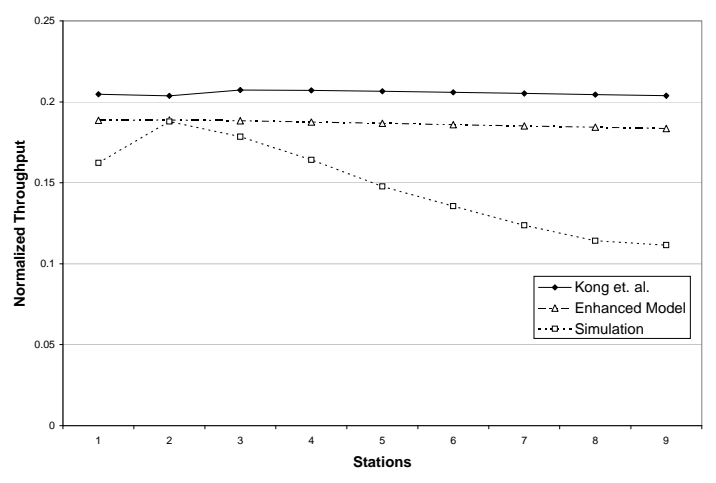

Figure 6: Comparing the Throughput of AC_VI

The model validation undertook by Kong et. al. shows a good performance of their model. The problem is that Kong et. al.'s simulation covers only 
stations where the only active access categories are $\mathrm{AC}_{-} \mathrm{VO}$ and $\mathrm{AC} \_\mathrm{BE}$, and with contention windows reducing the probability of virtual collision. The aim of this set of simulation is to compare the behavior of the enhanced model towards Kong et. al.'s model and simulation in scenarios where the stations have their three top priority access categories active using the default contention window ranges. Figure 5 and 6 show a comparison between the calculated throughputs of both $\mathrm{AC}_{-} \mathrm{VO}$ and $\mathrm{AC}_{-} \mathrm{VI}$ using both models and the results of simulations presented in previous section. Two main conclusions can be drawn from the graphs:

- first, both models give merely good results in cases with a low number of station, but are outperformed with a higher number of station $(M>3)$, this is due to a lack of exactitude of the real collision representation.

- In a low number of station case, the enhanced model's results are closer to the simulation results than Kong et. al. model's results. In a high number of station case, the effects of our enhancements are hidden by the real collision lack of exactitude.

In the following section, this issue is solved by deriving the real collision probability from measurements in order to give a better view of the global context.

\subsection{Measurement based use of the model}

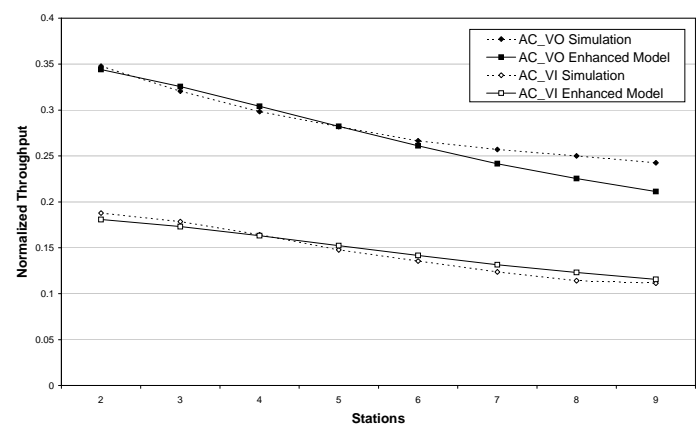

Figure 7: Comparing the Throughput of AC_VO and AC_VI with probability insertion

A set of calculations were made using the model inserting into it the mean real collision probability as recovered from the simulation (this being the ratio between total collisions and total access attempts). Figure 7 shows the throughputs as calculated by the model and the simulation. We can see that the results given by the model give a close view of the throughput achieved by each access category (as recovered from simulation results) even with a high number of station.

\section{CONCLUSION}

The paper presented a new Markov chain model of IEEE 802.11e EDCA. Its main contribution is it inherently represents the virtual collision phenomenon which was not precisely considered in previous models. In addition, the model corrects several backoff scheme related misconceptions of previous models. This made us introduce the concept of access attempt and differentiate it from an actual access. We claim this model to be an exhaustive model of the behavior of EDCA with respect to an access category in the saturation regime.

The model has been numerically solved and validated against simulation. It has shown to particularly fit the simulation when some of its parameters (collision probability, ...) are derived (typically by measurement) from network load. We believe that this is the best use of our model which can for instance find its application in the context of admission control for QoS support. More precisely, an access point could use our model to assess the maximum throughput that can be offered to one of its access categories by injecting into the model the collision probability observed on the medium. This is particularly one of our future directions in addition to reducing the model using Beizer rules (Beizer, 1971).

\section{REFERENCES}

802.11e (2005). IEEE Standard for Telecommunications and Information Exchange between Systems LAN/MAN specific Requirements - Part 11: Wireless LAN MAC and PHY specifications - Amendment 8: Medium Access Control QoS Enhancements.

Beizer, B. (1971). he architecture and Engineering of Digital Computer Complexes. Plenum Press, New York.

Bianchi, G. (2000). Performance analysis of ieee 802.11 distributed coordination function. IEEE Journal on selected areas in communications, 18(3):535-547.

Kong, Z., Tsang, D. H. K., Bensaou, B., and Gao, D. (2004). Performance analysis of ieee 802.11e contentionbased channel access. IEEE Journal on selected areas in communications, 22(10):2095-2106.

Mangold, S., Choi, S., Hiertz, G. R., Klein, O., and Walke, B. (2003). Analysis of ieee 802.11e for qos support in wireless lans. IEEE Wireless Communications, 10(6):40-50.

Wu, H., Wang, X., Zhang, Q., and Shen, X. (2006). Ieee 802.11e enhanced distributed channel access (edca) throughput analysis. IEEE International Conference on Communications, 1:223-228.

Zhu, H. and Chlamtac, I. (2005). Performance analysis for ieee $802.11 \mathrm{e}$ edcf service differentiation. IEEE Transactions on wireless Communications, 4(4):1779_ 1788. 